Article

\title{
Improved van Zyl Polarimetric Decomposition Lessening the Overestimation of Volume Scattering Power
}

Xiaoguang Cheng ${ }^{1, *}$, Wenli Huang ${ }^{2}$ and Jianya Gong ${ }^{1}$

1 State Key Laboratory of Information Engineering in Surveying, Mapping and Remote Sensing, Wuhan University, 129 Luoyu Road, Wuhan, Hubei 430079, China; E-Mail: gongjy@whu.edu.cn

2 Department of Geographical Sciences, University of Maryland, College Park, MD 20742, USA; E-Mails: hw1208@gmail.com

* Author to whom correspondence should be addressed; E-Mail: shenjianzi.cheng@gmail.com; Tel.: +86-134-1951-3742.

Received: 30 April 2014; in revised form: 19 June 2014 / Accepted: 27 June 2014 /

Published: 8 July 2014

\begin{abstract}
This paper improves van Zyl's Nonnegative Eigenvalue Decomposition (NNED). Orientation angle compensation and helix scattering are introduced to the decomposition. The volume scattering parameters that explain the most cross-polarized power are selected. If volume scattering and helix scattering explain all cross-polarized power in the measured coherency matrix, then simply perform van Zyl decomposition to the remainder matrix; otherwise, the measured coherency matrix is decomposed into three components, i.e., helix scattering, volume scattering, and one ground scattering. The latter two scattering are all modeled by Neumann's adaptive depolarizing model, according to which some cross-polarized power is attributed to ground scattering hence the orientation angle randomness of volume scattering and the dominant ground scattering are obtained. In this way, all cross-polarized power could be well explained. Experiments using UAVSAR data showed that more than $99.8 \%$ of total pixels are well fitted. Negative power is avoided. Compared with van Zyl decomposition, volume scattering power is reduced by up to $8.73 \%$ on average. The given volume scattering power is often lower than that by three latest NNED.
\end{abstract}

Keywords: polarimetric SAR; polarimetric decomposition; nonnegative eigenvalue decomposition; scattering model 


\section{Introduction}

In the field of polarimetric Synthetic Aperture Radar (PolSAR), model-based incoherent decomposition is an important research topic [1]. Since Freeman and Durden proposed the three-component decomposition in 1992 [2] and 1998 [3], more than 20 decompositions have been published [4-16]. Model-based decomposition has been successfully used in PolSAR image classification [17-21], speckle filtering [22], polarimetric SAR Interferometry [23], wetland research [24], soil moisture and roughness estimation [25-27], target detection [28,29], disaster assessment [30], and so on. In the past several years, the largest advances include adaptive scattering models [16,26,31,32], Orientation Angle Compensation (OAC) [33], Nonnegative Eigenvalue Constraint (NNEC) [7], etc.

van Zyl et al. [7] demonstrated that if model-based decomposition is valid, then after subtracting any components from the observed covariance or coherency matrix, the remainder matrix must be positive semidefinite, or its eigenvalues must be nonnegative. This constraint is named as NNEC. It could be easily proved that, usually, the decomposition results satisfy NNEC as long as the obtained component powers are nonnegative. In $[8,15]$, NNEC was adopted to eliminate negative power as much as possible. In recent years, several Nonnegative Eigenvalue Decompositions (NNED) were proposed by van Zyl et al. [7], Arii et al. [6], Cui et al. [12], and Wang et al. [13]. In all NNED, the maximum volume scattering power that makes the remainder matrix positive semidefinite is thought to be optimal and selected. In this way, the overestimation of volume scattering power in Yamaguchi decomposition or Freeman-Durden decomposition is largely eliminated. In addition, negative power is fundamentally avoided.

However, NNED still has two potential problems. The first problem is the overestimation of volume scattering power is not entirely eliminated. Since the maximum volume scattering power in theory is adopted, the overestimation is inevitable to a large degree. When applying adaptive volume scattering models, the overestimation is more serious [6,13].

The second problem is how to explain cross-polarized power. Subject to the Reflection Symmetry Assumption (RSA) and employment of elemental scatterers of which cross-polarized complex scattering coefficient, or $S_{H V}$ is zero, the derived coherent models of surface scattering and double-bounce scattering cannot describe depolarizing effect. However, it was observed [7] that if volume scattering power is computed with RSA, then, in many natural forest pixels, volume scattering and helix scattering cannot explain all cross-polarized power. van Zyl attributed these unexplained cross-polarized power to a remainder component which is thought to represent terrain effects and rough surface scattering. If volume scattering power is computed without RSA, then in almost every pixel, volume scattering and helix scattering cannot explain all cross-polarized power. Wang et al. [13] and Cui et al. [12] utilized elemental scatterers of which $S_{H V}$ is non-zero and coherent models, to explain these remaining cross-polarized power. Unfortunately, among all widely recognized scattering mechanisms, only helix scattering with standard orientation has non-zero $S_{H V}$.

Some researchers may argue that there are possibly some unknown scattering mechanisms, which could produce cross-polarized power. However, according to the latest incoherent adaptive scattering models, such as X-Bragg model [26], Arii's model [31], and Neumann's model [32], when the orientation angles of scatterers in one component are not exactly the same, this component will give cross-polarized power as long as $S_{H H}+S_{V V} \neq 0$, where $S_{H H}$ and $S_{V V}$ are the complex scattering 
coefficients, $\mathrm{HH}$ means horizontal transmitting and horizontal receiving, VV means vertical transmitting and vertical receiving.

Generally, in incoherent scattering, the assumption that orientation angles in one component are the same cannot be guaranteed even for man-made targets, let alone for natural distributed targets. Natural terrain, like the ground in old-growth forest, is usually rough even when the wavelength is long. Hence, surface scattering could produce non-zero cross-polarized power. Similarly, double-bounce scattering with cross-polarized power also widely distribute in natural environment. From this perspective, both surface scattering and double-bounce scattering could give cross-polarized power in incoherent scattering. Neumann [32] found that in forest, ground scattering has significant cross-polarized power. Therefore, it is wiser to apply incoherent models for ground scattering to explain cross-polarized power instead of unknown scattering mechanisms. The above idea is also supported in [10,15,34]. Singh et al. [10] pointed out that, to understand the depolarization effects on the decomposition results, using extended incoherent ground scattering models are required. Lee et al. [15] tried to incorporate incoherent ground scattering models into Freeman-Durden decomposition.

A new decomposition was proposed in this paper. Since RSA is applied in the computation of volume scattering, the proposed decomposition could be considered as an improved version of van Zyl decomposition. Experiment using Uninhabited Aerial Vehicle Synthetic Aperture Radar (UAVSAR) data well demonstrated the effectiveness of the proposed decomposition.

\section{Scattering Models}

In this paper, $T_{m n}$ stands for the element in the $m_{t h}$ row and $n_{t h}$ column of matrix [T].

\subsection{Helix Scattering Model}

The helix scattering model $\left[T_{H}\right]$ can be found in [4], so the authors would not give it here.

\subsection{Volume Scattering Model}

In [35], Neumann et al. assume the scattering matrix of an elemental scatterer is:

$$
[S]=\left[\begin{array}{cc}
S_{H H} & 0 \\
0 & S_{V V}
\end{array}\right]
$$

If the orientation angles of elemental scatterer follow unimodal circular normal von Mises distribution and mean orientation angle is zero, the derived reflection symmetric scattering model $\left[T_{\text {Neum }}\right]$ will be:

$$
\left[T_{\text {Neum }}\right]=\frac{1}{L+N}\left[T_{\text {Neumann }}\right]
$$

with

$$
\begin{gathered}
{\left[T_{\text {Neumann }}\right]=\left[\begin{array}{ccc}
L & g_{c}(\tau) M & 0 \\
g_{c}(\tau) M^{*} & (1+g(\tau)) N / 2 & 0 \\
0 & 0 & (1-g(\tau)) N / 2
\end{array}\right]} \\
L=\left|S_{H H}+S_{V V}\right|^{2}, M=\left(S_{H H}+S_{V V}\right)\left(S_{H H}-S_{V V}\right)^{*}, N=\left|S_{H H}-S_{V V}\right|^{2}
\end{gathered}
$$




$$
\tau=I_{0}(k) \mathrm{e}^{-k}, g_{c}(\tau)=\frac{I_{1}(k)}{I_{0}(k)}, g(\tau)=\frac{I_{2}(k)}{I_{0}(k)}
$$

$*$ is the sign of complex conjugate operator; $\tau$, as the randomness of orientation angles, is defined in the range of $[0,1] ; k$ is the concentration degree of orientation angles; $I_{n}(k)$ is the modified Bessel function of order $n$ and parameter $k$; and $g(\tau) \in[0,1], g_{c}(\tau) \in[0,1] .\left[T_{\text {Neum }}\right]$ describes coherent scattering if $\tau=0$. The advantages of $\left[T_{\text {Neum }}\right]$ over X-Bragg model and improved Yamaguchi's model were clearly pointed out by Lee et al. [15].

When horizontal dipole (see Equation (6) for scattering matrix $\left[S_{H o r z}\right]$ ) is used as elemental scatterer, the volume scattering model is $\left[T_{V o l_{-}}\right]$(see Equation (8)); when vertical dipole (see Equation (7) for scattering matrix $\left[S_{\text {Vert }}\right]$ ) is used as elemental scatterer, the volume scattering model is $\left[T_{V o l \_}\right]$(see Equation (9)). $\tau_{V}$ is the $\tau$ of volume scattering.

$$
\begin{gathered}
{\left[S_{\text {Horz }}\right]=\left[\begin{array}{ll}
1 & 0 \\
0 & 0
\end{array}\right]} \\
{\left[S_{\text {Vert }}\right]=\left[\begin{array}{ll}
0 & 0 \\
0 & 1
\end{array}\right]} \\
{\left[T_{V o l_{-} H}\right]=\frac{1}{2}\left[\begin{array}{ccc}
1 & g_{c}\left(\tau_{V}\right) & 0 \\
g_{c}\left(\tau_{V}\right) & \frac{1+g\left(\tau_{V}\right)}{2} & 0 \\
0 & 0 & \frac{1-g\left(\tau_{V}\right)}{2}
\end{array}\right]} \\
{\left[T_{V o l_{-} V}\right]=\frac{1}{2}\left[\begin{array}{ccc}
1 & -g_{c}\left(\tau_{V}\right) & 0 \\
-g_{c}\left(\tau_{V}\right) & \frac{1+g\left(\tau_{V}\right)}{2} & 0 \\
0 & 0 & \frac{1-g\left(\tau_{V}\right)}{2}
\end{array}\right]}
\end{gathered}
$$

\subsection{Ground Scattering Model}

The classical coherent surface scattering model is:

$$
\left[T_{S}\right]=\frac{1}{1+|\beta|^{2}}\left[\begin{array}{ccc}
1 & \beta^{*} & 0 \\
\beta & |\beta|^{2} & 0 \\
0 & 0 & 0
\end{array}\right]
$$

The classical coherent double-bounce scattering model is:

$$
\left[T_{D}\right]=\frac{1}{1+|\alpha|^{2}}\left[\begin{array}{ccc}
|\alpha|^{2} & \alpha & 0 \\
\alpha^{*} & 1 & 0 \\
0 & 0 & 0
\end{array}\right]
$$

In case the cross-polarized power cannot be entirely explained by helix scattering and volume scattering, the dominant ground scattering is described by $\left[T_{\text {Neum }}\right]$ to explain a proportion of cross-polarized power. Although $\left[T_{\text {Neum }}\right]$ was originally proposed to describe volume scattering, as a generic scattering model, $\left[T_{\text {Neum }}\right]$ is also capable of describing ground scattering by using proper scattering matrix of elemental scatterers [36,37]. For example, combining the complex scattering coefficients predicted by Bragg model [26] with von Mises distribution, the derived incoherent model could describe depolarizing effects, and it may be considered as another X-Bragg model [26]. When 
[ $\left.T_{\text {Neum }}\right]$ is used to describe surface scattering, the $\tau$ is denoted as $\tau_{S}$; when $\left[T_{\text {Neum }}\right]$ is used to describe double-bounce scattering, the $\tau$ is denoted as $\tau_{D}$.

\section{Polarimetric Decomposition}

Figure 1 is the flow chart of the proposed decomposition.

Figure 1. The flow chart of the proposed decomposition.

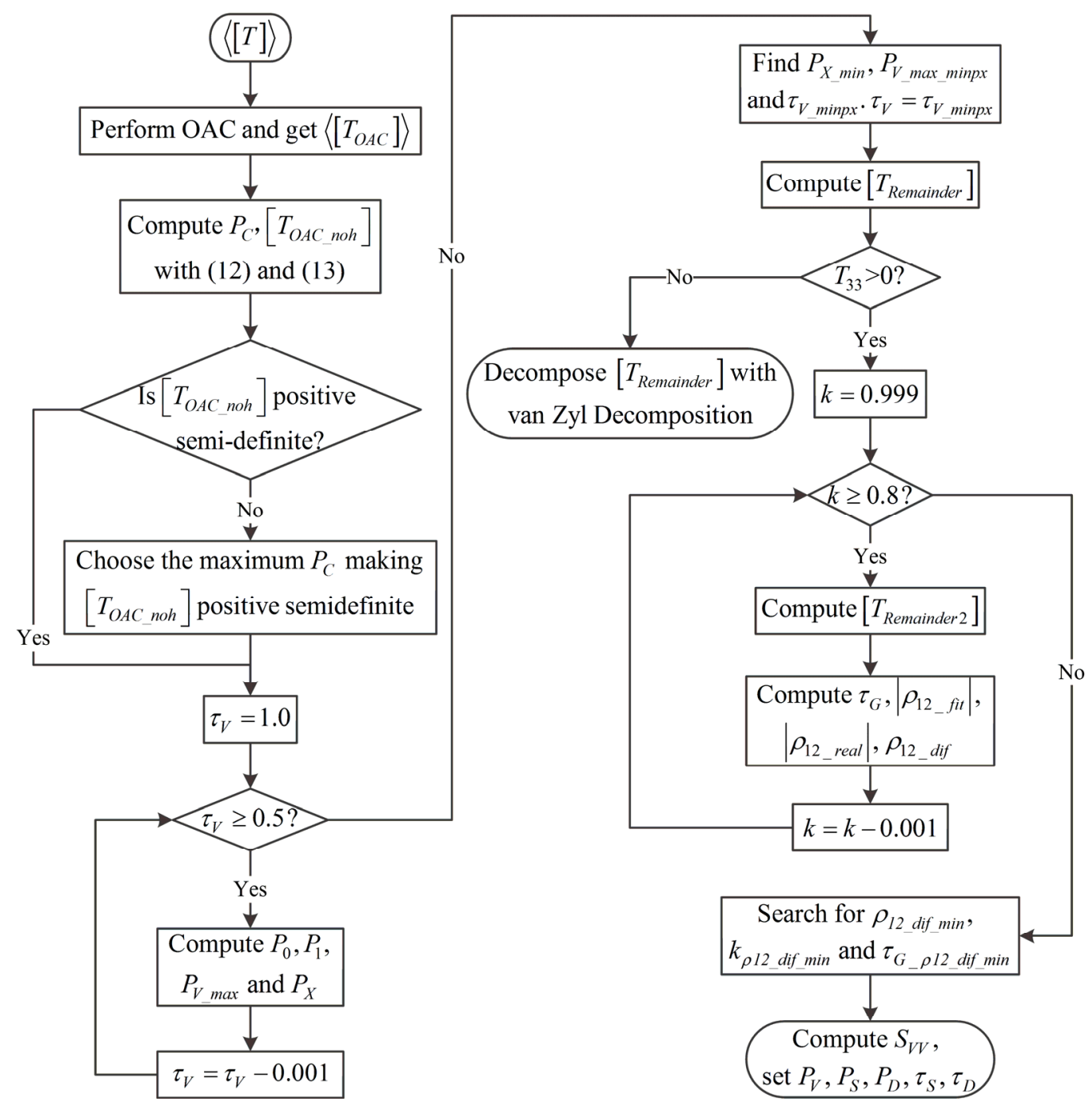

\subsection{Orientation Angle Compensation}

In order to make $\langle[T]\rangle$ be closer to RSA so that it is more reasonable to utilize $\left[T_{N e u m}\right]$, perform OAC to $\langle[T]\rangle$ and get $\left\langle\left[T_{O A C}\right]\right\rangle$. The details of OAC processing could be found in [33]. \langle\rangle stands for ensemble average processing.

\subsection{Helix Scattering Power Computation}

Helix scattering power $P_{C}$ is first calculated as [4]:

$$
P_{C}=2\left|\operatorname{Im}\left(\left\langle T_{23}\right\rangle\right)\right|
$$


$\operatorname{Re}(x)$ and $\operatorname{Im}(x)$ denote the real and imaginary part of complex number $x$ in this paper. Subtracting helix scattering from $\left\langle\left[T_{O A C}\right]\right\rangle$, we have:

$$
\left[T_{O A C_{-} n o h}\right]=\left\langle\left[T_{O A C}\right]\right\rangle-P_{C}\left[T_{H}\right]
$$

However, $P_{C}$ given in Equation (12) cannot always guarantee $\left[T_{O A C \_n o h}\right]$ satisfy NNEC. Once $\left[T_{\text {OAC_noh }}\right]$ violates NNEC, $P_{C}$ is recomputed as the maximum value that makes $\left[T_{O A C_{-} n o h}\right]$ positive semidefinite. In this paper, $T_{13}$ in $\left[T_{O A C \_n o h}\right]$ is ignored and treated to be zero because no scattering model could explain non-zero $T_{13}$. Since, in most cases, $\left[T_{O A C_{-} n o h}\right]$ has $T_{23}=0$, so $\left[T_{O A C_{-} n o h}\right]$ is reflection symmetric.

\subsection{Volume Scattering Parameter Computation}

In [7], van Zyl et al. gave the formulas of computing volume scattering power $P_{V}$ with RSA. Wang et al. [13] and Cui et al. [12] extended the formulas without RSA. Assume $\left[T_{O A C \_n o h}\right]$ is:

$$
\left[T_{\text {OAC_noh }}\right]=\left[\begin{array}{ccc}
A_{11} & A_{12} & 0 \\
A_{12}^{*} & A_{22} & 0 \\
0 & 0 & A_{33}
\end{array}\right]
$$

Assume the volume scattering model $\left[T_{V}\right]$ is:

$$
\left[T_{V}\right]=\left[\begin{array}{ccc}
B_{11} & B_{12} & 0 \\
B_{12}^{*} & B_{22} & 0 \\
0 & 0 & B_{33}
\end{array}\right]
$$

Subtracting volume scattering from $\left[T_{O A C_{-} n o h}\right]$, the remainder matrix $\left[T_{\text {Remainder }}\right]$ is:

$$
\left[T_{\text {Remainder }}\right]=\left[T_{O A C \_n o h}\right]-P_{V}\left[T_{V}\right]
$$

In $[6,7,12,13]$, the maximum $P_{V}$ (denoted as $\left.P_{V_{-} \text {max }}\right)$ that makes $\left[T_{\text {Remainder }}\right]$ positive semidefinite is selected, so the smallest eigenvalue of $\left[T_{\text {Remainder }}\right]$ is zero. Therefore, we have:

$$
\left|\left[T_{\text {Remainder }}\right]\right|=\left|\left[T_{O A C_{-} \text {noh }}\right]-P_{V_{-} \max }\left[T_{V}\right]\right|=0
$$

$|[T]|$ is the determinant of $[T]$. If $\left[T_{V}\right]$ is strictly positive definite (nearly all available volume scattering models reach this demand), then Equation (17) could be rewritten as:

$$
\left|\left[T_{V}\right]^{-1}\left[T_{O A C \_n o h}\right]-P_{V \_ \text {max }} I\right|=0
$$

where $I$ is a $3 \times 3$ identity matrix. From Equation (18), we could know $P_{V_{-}} \max$ is the smallest eigenvalue of $\left[T_{V}\right]^{-1}\left[T_{O A C_{-} n o h}\right]$. $P_{V_{-} \max }$ could be written as another form:

$$
P_{V_{-} \max }=\max (W) \text { with } W=\left\{P_{V} \mid 0 \leq P_{V} \leq \min \left\{P_{0}, P_{1}\right\}\right\}
$$

$P_{1}=A_{33} / B_{33}, P_{0}$ is the smallest nonnegative solution of Equation (20), and $\min \left\{P_{0}, P_{1}\right\}$ means the smaller one of $P_{0}$ and $P_{1}$.

$$
\left(A_{11}-P_{V} B_{11}\right)\left(A_{22}-P_{V} B_{22}\right)-\left|A_{12}-P_{V} B_{12}\right|^{2}=0
$$

For adaptive volume scattering models, the parameters that give the maximum $P_{V_{-} \text {max }}$ (denoted as $\left.P_{V_{-} \text {max _maxpv }}\right)$ are selected. The above criterion is named as 'maximum $P_{V}$ criterion.' It was proved by Cheng [38] that if $\operatorname{Re}\left(T_{12}\right)$ in $\left[T_{O A C_{-} n o h}\right]$ is positive, then $P_{V_{-} \max }$ given by $\left[T_{V o l_{-} H}\left(\tau_{V}\right)\right]$ is larger than that by $\left[T_{V o l \_V}\left(\tau_{V}\right)\right]$; if $\operatorname{Re}\left(T_{12}\right)$ in $\left[T_{O A C_{-} n o h}\right]$ is negative, then $P_{V_{-} m a x}$ given by $\left[T_{V o l_{-} V}\left(\tau_{V}\right)\right]$ is 
larger than that by $\left[T_{V o l \_}\left(\tau_{V}\right)\right]$. Thus, with maximum $P_{V}$ criterion, if $\operatorname{Re}\left(T_{12}\right)>0$ in $\left[T_{O A C \_n o h}\right]$, then we should use $\left[T_{V o l_{-} H}\right]$; if $\operatorname{Re}\left(T_{12}\right)<0$ in $\left[T_{O A C_{-} n o h}\right]$, then we should use $\left[T_{V o l \_V}\right]$. Here, we denote the selected model as $\left[T_{V o l}\right]$. Obviously, $\tau_{V}$ is the only parameter in $\left[T_{V o l}\right]$. We denote the $\tau_{V}$ that gives $P_{V_{-} \text {max _maxpv }}$ as $\tau_{V_{-} \text {maxpv }}$. It is well known that volume scattering is regarded to be primarily from trees. The observation in [35] indicated the $\tau$ of forest mainly lies in $[0.6,0.9]$, so $\tau_{V}$ is confined within $[0.5,1.0]$.

Evidently, maximum $P_{V}$ criterion tends to overestimates real $P_{V}$ since the maximum $P_{V}$ in theory is selected. We need a new criterion. It is widely recognized that cross-polarized power mainly or even entirely comes from volume scattering [3-5,8,10,14]. From this perspective, we could let volume scattering explain as much cross-polarized power as possible. The cross-polarized power not explained by volume scattering and helix scattering, namely, $P_{X}$, is expressed as:

$$
P_{X}=F_{33}=A_{33}-P_{V} B_{33}
$$

$F_{33}$ stands for $T_{33}$ of $\left[T_{\text {Remainder }}\right]$. From Equation (21), we could know, when $B_{33}$ is fixed, then the larger $P_{V}$, the smaller $P_{X}$. In other words, when we use a fixed $\left[T_{V}\right]$, the maximum $P_{V}$ constrained by NNEC lets volume scattering explain the most cross-polarized power. However, for adaptive models, like $\left[T_{V o l}\right]$, we cannot guarantee the maximum $P_{V}$ yields the minimum $P_{X}$ because of the varying $B_{33}$. Here the volume scattering parameters that produce the minimum $P_{X}$ (denoted as $\left.P_{X_{-} \text {min }}\right)$ are selected. This new criterion is called 'minimum $P_{X}$ criterion.'

Again, $\tau_{V}$ is confined within $[0.5,1.0]$. For each $\tau_{V}$, we compute $\left[T_{V o l}\left(\tau_{V}\right)\right]$ and corresponding $P_{V_{-} \max }, P_{X}$. Among all results, choose the $\tau_{V}$ and $P_{V_{-} \max }$ that produce $P_{X_{-} \text {min }}$. If more than one combinations of $\tau_{V}$ and $P_{V_{-} \max }$ give the same $P_{X_{-} \text {min }}$, then choose the one with the smallest $P_{V_{-} \max }$. We denote the chosen $\tau_{V}$ as $\tau_{V_{-} \operatorname{minpx}}$ and $P_{V_{-} \text {max }}$ as $P_{V_{-} \text {max }}$ minpx . Considering that $P_{V_{-} m a x \_m a x p v}$ is the maximal one of $P_{V_{-} \text {max }}$, we have $P_{V_{-} \max \_ \text {minpx }} \leq P_{V_{-} \text {max } \_ \text {maxpv }}$, indicating that minimum $P_{X}$ criterion tends to lower the estimation of $P_{V}$ (see Figure 2). Combining this conclusion with Equation (21), we could know that in order to get smaller $P_{X}, P_{V} B_{33}$ needs to be larger. Since $P_{V_{-} \text {max } \_ \text {minpx }} \leq P_{V_{-} \text {max _maxpv }}$, so $B_{33}$ or $\tau_{V}$ by minimum $P_{X}$ criterion should be larger than $B_{33}$ or $\tau_{V}$ by maximum $P_{V}$ criterion. In other words, $\tau_{V_{-} \text {minpx }}>\tau_{V_{-} \text {maxpv }}$. Section 5 provides a detailed analysis. Finally, $\left[T_{\text {Remainder }}\right]$ is expressed as:

$$
\left[T_{\text {Remainder }}\right]=\left[T_{O A C_{-} n o h}\right]-P_{V_{-} \max \_\operatorname{minpx}}\left[T_{V o l}\left(\tau_{V_{-} \operatorname{minpx}}\right)\right]
$$

Figure 2. The difference between minimum $P_{X}$ criterion and maximum $P_{V}$ criterion.

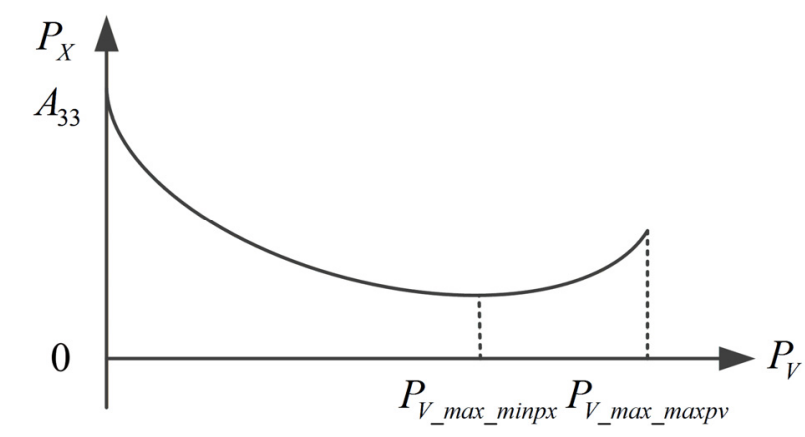




\subsection{Ground Scattering Solution}

If $T_{33}=0$ in $\left[T_{\text {Remainder }}\right]$, or volume scattering and helix scattering explain all cross-polarized power in $\langle[T]\rangle$, then with Eigen-decomposition, we could decompose $\left[T_{\text {Remainder }}\right]$ into two components of which Pauli-basis target vectors are orthogonal to each other. The correspondence between these eigenvectors and scattering mechanisms could be determined using van Zyl's method [7]. $\tau_{S}=0$ and $\tau_{D}=0$. The whole decomposition could be written as:

$$
\left\langle\left[T_{O A C}\right]\right\rangle=P_{C}\left[T_{H}\right]+P_{V_{-} m a x \_m i n p x}\left[T_{V o l}\left(\tau_{V \_ \text {minpx }}\right)\right]+P_{D}\left[T_{D}\right]+P_{S}\left[T_{S}\right]
$$

If $T_{33}>0$ in $\left[T_{\text {Remainder }}\right]$, one ground scattering is modeled by $\left[T_{\text {Neum }}\right]$ to explain some cross-polarized power in $\left[T_{\text {OAC_noh }}\right]$. The next step is determining which ground scattering should be modeled by $\left[T_{\text {Neum }}\right]$. It was pointed out in [36] that, usually, in surface scattering model, $T_{11}>T_{22}+T_{33}$; in volume scattering model, $T_{11}=T_{22}+T_{33}$; in double bounce scattering model, $T_{11}<T_{22}+T_{33}$. Taken in this sense, we give the following judging criterion: if $A_{11}>A_{22}+A_{33}$, then surface scattering is the dominant ground scattering, so we model it with $\left[T_{\text {Neum }}\right]$, and $\left[T_{\text {OAC_noh }}\right]$ is written as Equation (24); if $A_{11}<A_{22}+A_{33}$, then double bounce scattering is the dominant ground scattering, so we model it with $\left[T_{\text {Neum }}\right]$, and $\left[T_{O A C_{-} n o h}\right]$ is written as Equation (25).

$$
\begin{gathered}
{\left[T_{\text {OAC_noh }}\right]=2 f_{V}\left[T_{V o l}\left(\tau_{V \_ \text {minpx }}\right)\right]+f_{S}\left[T_{\text {Neumann }}\left(\tau_{S}\right)\right]} \\
{\left[T_{\text {OAC_noh }}\right]=2 f_{V}\left[T_{V o l}\left(\tau_{V \_ \text {minpx }}\right)\right]+f_{D}\left[T_{\text {Neumann }}\left(\tau_{D}\right)\right]}
\end{gathered}
$$

In Equations (21) and (22), the coefficients $f_{V}, f_{S}$, and $f_{D}$ are introduced to make the decomposition expression look simpler in form. After doing some mathematical operations to Equation (24) or Equation (25), we have:

$$
\begin{gathered}
g\left(\tau_{G}\right)=\frac{A_{22}-A_{33}-f_{V} g\left(\tau_{V \_ \text {minpx }}\right)}{A_{22}+A_{33}-f_{V}} \\
\frac{1+g\left(\tau_{G}\right)}{g_{c}^{2}\left(\tau_{G}\right)}=\frac{\left(A_{11}-f_{V}\right)\left(2 A_{22}-f_{V}\left(1+g\left(\tau_{V \_ \text {minp }}\right)\right)\right)}{\left|A_{12} \pm f_{V} g_{C}\left(\tau_{V \_ \text {minpx }}\right)\right|^{2}}
\end{gathered}
$$

$\tau_{G}$ denotes $\tau_{S}$ in Equation (24) or $\tau_{D}$ in Equation (25). To the right of the " $=$ " sign in Equation (27), the " \pm " sign depends on which volume scattering model is used. If we use $\left[T_{V o l_{-} H}\right]$, then "-" should be used; otherwise, "+" should be used. Two unknowns exist in Equations (26) and (27), namely, $\tau_{G}$ and $f_{V}$. In theory, we could get analytical solutions from Equations (26) and (27). But that is difficult due to the complexity of $g\left(\tau_{G}\right)$ and $g_{c}\left(\tau_{G}\right)$.

This problem may be solved from another point of view. $P_{V_{-} \max \_\operatorname{minpx}}$ still possibly overestimates volume scattering power because volume scattering is probably unable to explain so much cross-polarized power. If we lower $P_{V}$ to a value smaller than $P_{V_{-} \text {max }}$ minpx , we could check whether the new remainder matrix $\left[T_{\text {Remainder } 2}\right]$, which has positive cross-polarized power, could be well fitted with $\left[T_{\text {Neum }}\right]$ or not. Assume $P_{V}=P_{V_{-} m a x \_m i n p x} k$, where $k \in(0,1)$, then $\left[T_{\text {Remainder } 2}\right]$ is:

$$
\left[T_{\text {Remainder } 2}\right]=\left[T_{O A C_{-} n o h}\right]-P_{V_{-} \text {max _minpx }} k\left[T_{V o l}\left(\tau_{V_{-}} \text {minpx }\right)\right]
$$

To fit $\left[T_{\text {Remainder } 2}\right]$ with $\left[T_{\text {Neum }}\right]$, we relate them together in the following way: 


$$
\begin{aligned}
{\left[T_{\text {Remainder } 2}\right] } & =\left[\begin{array}{ccc}
G_{11} & G_{12} & 0 \\
G_{12}^{*} & G_{22} & 0 \\
0 & 0 & G_{33}
\end{array}\right] \\
& =\frac{G_{11}+G_{22}+G_{33}}{L+N}\left[\begin{array}{ccc}
L & g_{c}\left(\tau_{G}\right) M & 0 \\
g_{c}\left(\tau_{G}\right) M^{*} & \frac{1+g\left(\tau_{G}\right)}{2} N & 0 \\
0 & 0 & \frac{1-g\left(\tau_{G}\right)}{2} N
\end{array}\right]
\end{aligned}
$$

From Equation (29), we first obtain $\tau_{G}$ using:

$$
g\left(\tau_{G}\right)=\frac{G_{22}-G_{33}}{G_{22}+G_{33}}
$$

In $\left[T_{\text {Neum }}\right]$, the absolute value of the correlation coefficient between $T_{11}$ and $T_{22}$, namely, $\left|\rho_{12_{-} \text {fit }}\right|$, is:

$$
\left|\rho_{12_{-} f i t}\right|=\frac{\sqrt{2} g_{C}\left(\tau_{G}\right)}{\sqrt{1+g\left(\tau_{G}\right)}}
$$

The corresponding value in $\left[T_{\text {Remainder } 2}\right]$, namely, $\left|\rho_{12 \_r e a l}\right|$, is:

$$
\left|\rho_{12 \_ \text {real }}\right|=\frac{\left|G_{12}\right|}{\sqrt{G_{11} G_{22}}}
$$

If $\left[T_{\text {Remainder } 2}\right]$ is perfectly fitted with $\left[T_{\text {Neum }}\right]$, then $\left|\rho_{12 \_ \text {real }}\right|$ should be equal to $\left|\rho_{12_{-} f i t}\right|$. A simple measure $\rho_{12 \_d i f}$ is defined to measure the goodness of fitting $\left[T_{\text {Remainder } 2}\right]$ with $k$ and $\tau_{G}$.

$$
\rho_{12 \_d i f}=|| \rho_{12 \_f i t}|-| \rho_{12 \_ \text {real }}||
$$

The $k$ and $\tau_{G}$ yielding the minimum $\rho_{12 \_d i f}$, namely, $\rho_{12 \_d i f \_m i n}$, are thought to be the best fit. Here, we refer to them as $k_{\rho 12_{-} d i f_{-} m i n}$ and $\tau_{G_{-} \rho 12_{-} d i f \_m i n}$. If $\rho_{12 \_d i f \_m i n}=0, k_{\rho 12_{\_} d i f_{\_} \text {min }}$ and $\tau_{G_{-} \rho 12_{-} \text {dif_min }}$ are the perfect parameters to fit $\left[T_{\text {Remainder } 2}\right]$ with $\left[T_{\text {Neum }}\right]$.

With $k_{\rho 12_{-} d i f \_m i n}$ and $\tau_{G_{-} \rho 12_{-} d i f_{-} m i n}$, we could further get the value of $S_{V V}, L, M$, and $N$ (see Appendix). Finally, the solution of Equation (24) is:

$$
\begin{gathered}
P_{V}=P_{V_{-} \text {max } \_ \text {minpx }} k_{\rho 12_{-} \text {dif_min }}, P_{S}=G_{11}+G_{22}+G_{33}-P_{V} \\
\tau_{S}=\tau_{G_{-} \rho 12 \_d i f \_m i n}, P_{D}=0, \tau_{D}=0
\end{gathered}
$$

The solution of Equation (25) is:

$$
\begin{gathered}
P_{V}=P_{V \_ \text {max } \_ \text {minpx }} k_{\rho 12 \_d i f \_ \text {min }}, P_{D}=G_{11}+G_{22}+G_{33}-P_{V} \\
\tau_{D}=\tau_{G \_\rho 12 \_d i f \_m i n}, P_{S}=0, \tau_{S}=0
\end{gathered}
$$

We could easily know, $P_{V}<P_{V_{-} m a x \_m i n p x}$. Experiment reveals that the pixels whose $T_{33}>0$ in $\left[T_{\text {Remainder }}\right]$ are primarily located in forest where volume scattering dominates. To avoid the searching of $\rho_{12 \_d i f \_m i n}$ ending up in a small $k$ so that volume scattering is not dominant in the decomposition results, the varying of $k$ is empirically confined in $[0.8,1.0)$. From above derivation, we could know negative power is completely avoided. 


\section{Experiment}

UAVSAR is a fully polarimetric L-band sensor designed for acquiring airborne repeat-track interferometry SAR data [39]. Its applications include monitoring ground deformations, ice dynamics, volcano dynamics, local sea ice dynamics, time-varying evaporation and hydraulic properties of soils, and aboveground biomass [40]. UAVSAR data collected near Howland Forest, Maine, USA, on 5 August 2009 under a clear weather was used to test the applicability of proposed method. The study site is relatively flat and consists of forests, bare land, rivers, wetlands, road, buildings, etc. The data was downloaded from Alaska Satellite Facility website [41]. The look angle range is approximately $\left[25^{\circ}, 65^{\circ}\right]$, while the local incidence angles vary within $\left[0^{\circ}, 90^{\circ}\right]$. Basic scattering area correction, antenna pattern correction and range dependent radiometric correction have been performed. The resolution of the ground range image is $5 \mathrm{~m}$. Lee sigma filtering [42] is implemented in a $9 \times 9$ window. The equivalent number of looks is hard to estimate for ground range image because of lack of single look data. However, in multi-look slant range image, ensemble averaging was implemented with 12 looks in azimuth direction and three looks in range direction.

Among all pixels, $99.83 \%$ are perfectly fitted and only $0.17 \%$ cannot get good fitting of $T_{12}$. Figure $3 \mathrm{c}$ is the image of component power normalized by $P_{\text {span }}$, where $P_{\text {span }}$ is the span of $\langle[T]\rangle$. Figure $3 \mathrm{~d}$ is the image of $\tau$ of different components.

From Figure 3c, we could see all the major land cover features are identified. Dense natural forests are colored with bright green, indicating $P_{V}$ is large. A large proportion of dense natural forests are characterized by non-zero $P_{D}$ and zero $P_{S}$, although not all. $\tau_{V}$ mostly concentrates in $[0.60,0.90]$. Non-zero $\tau_{D}$ primarily locates in dense natural forests with value in $[0.03,0.35]$. Only a small number of forest pixels show non-zero $\tau_{S}$. It is found that they mainly lie in the boundaries between forests and land with little vegetation cover. However, in forests with low canopy density, the pixels may have $\tau_{S}=0$ and $\tau_{D}=0$. In the upper right corner of the image, there exists a sparse forest. This area gets much higher proportion of $P_{S}$ compared with dense forests. Since the tree cover is low, it is reasonable to have more surface scattering from ground. In most locations of this sparse forest, $\tau_{V}=1$, $\tau_{S}=0$, and $\tau_{D}=0$, meaning volume scattering and helix scattering do explain all cross-polarized power. We could observe from Figure $3 d$ that, generally, in forest, the easier to be accessed by human beings or the lower canopy density, the more likely to have $\tau_{D}=0$. We may interpret this phenomenon in the following way: in easily accessible forests, the terrain may be relatively flat and the understory may probably be underdeveloped; in the untraversed forests, the understory is fully developed, making the scattering process very complex. Most of the areas dominated by surface scattering, like river surfaces, airport, and grasslands, are colored with blue, showing that $P_{S}$ is quite high. In these areas, $P_{S}$ and $P_{D}$ are obtained with van Zyl's method, thus, $\tau_{S}=0$ and $\tau_{D}=0$. In urban areas, the main buildings are oriented parallel to SAR azimuth direction. Many pixels near buildings are characterized by high $P_{D}$ while a small proportion show high $P_{S}$. An apparent characteristic of these areas dominated by surface scattering or double bounce scattering is, they almost all have $\tau_{V}=1, \tau_{S}=0$, and $\tau_{D}=0$, which differs greatly from the dense natural forests.

The results of the proposed decomposition were compared with these of van Zyl decomposition and the other three latest NNED raised by Cui et al. [12] and Wang et al. [13]. Two NNED were raised by Cui et al. [12] and they only differ in the decomposition of the remainder matrix, one is based on 
Eigen-decomposition, namely, Cui1, and the other on model fitting, namely, Cui2. It is found that the performance of Cuil is not as good as Cui2 and Wang decomposition, while the results of Cui2 and Wang decomposition are quite similar. To avoid five profiles existing in one plot so the readers are confused, Figure 4 only gives the profiles of Wang, van Zyl and the proposed decomposition along three 500-m-long lines in Figure 3b. Red, green, and blue lines cover natural forests, airport, and urban areas, respectively.

Figure 3. (a) Optical images from National Agriculture Imagery Program (short for NAIP and administered by USDA-FSA Aerial Photography Field Office) 2009; (b) Pauli color-coded image; (c) Image composed of different components power, red is for $P_{D} / P_{\text {span }}$, green is for $P_{V} / P_{\text {span }}$, and blue is for $P_{S} / P_{\text {span }} ;(\mathbf{d})$ Image composed of $\tau$ of different components, red is for $\tau_{D}$, green is for $\tau_{V}$, and blue is for $\tau_{S}$. The geographic coordinate of image center is $68.656^{\circ} \mathrm{W}, 44.943^{\circ} \mathrm{N}$.

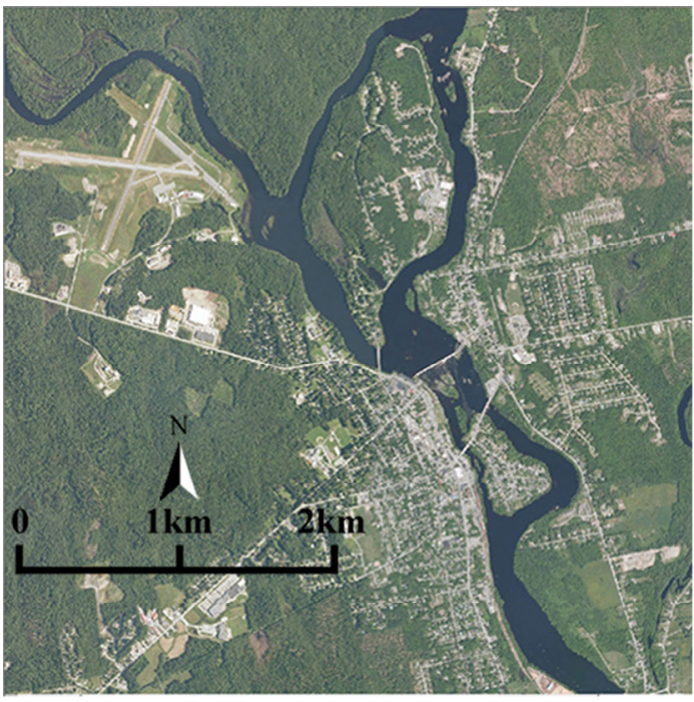

(a)

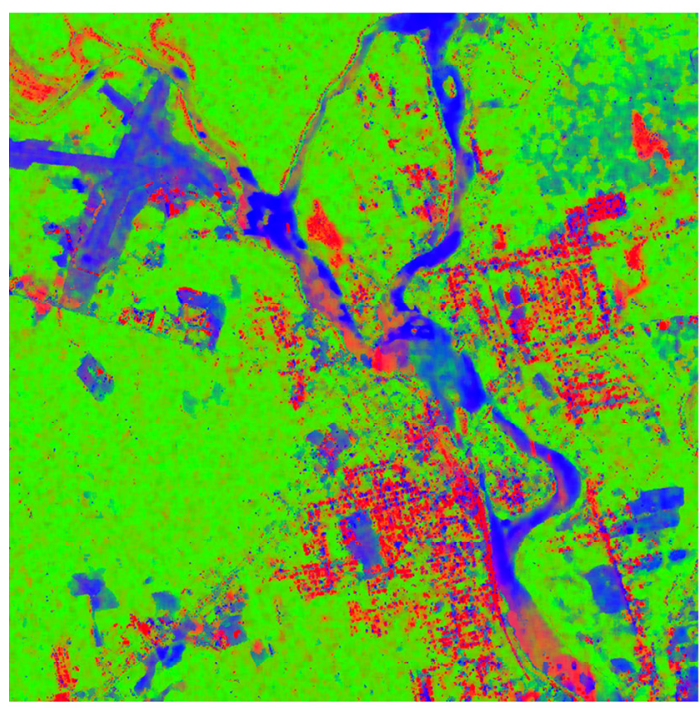

(c)

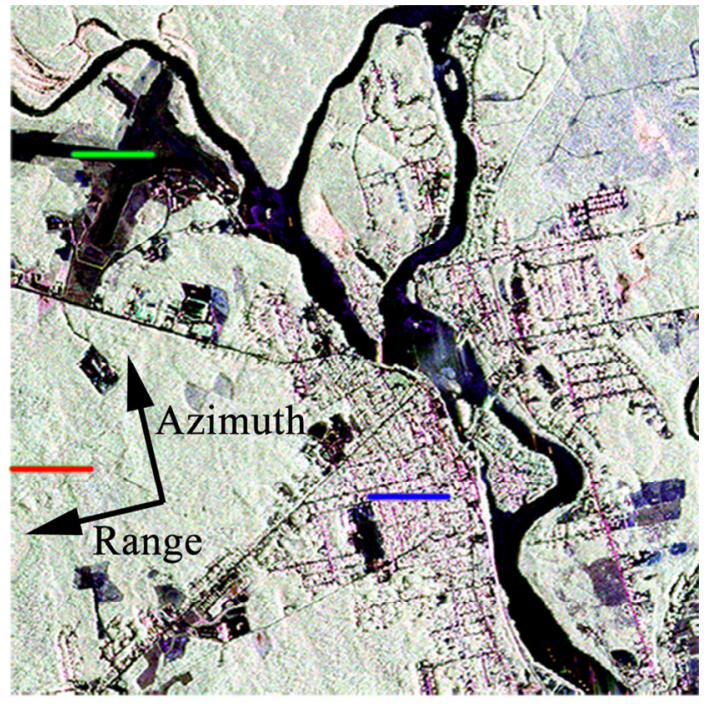

(b)

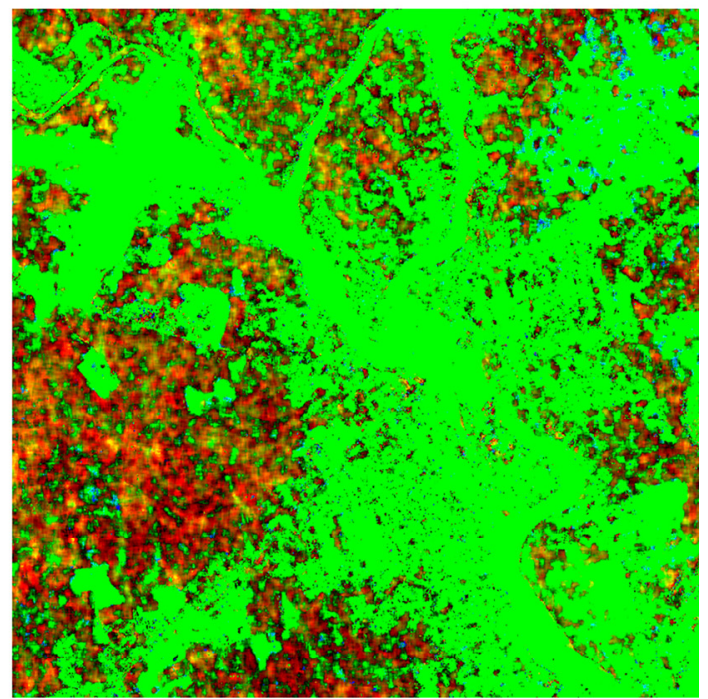

(d) 
Compared with van Zyl decomposition with OAC, the proposed decomposition lowered the estimation of $P_{V}$ in all pixels. The degree is $-7.72 \%$ on average and the standard deviation is 0.103 . In airports and almost all building areas, the proposed method gives the lowest estimation of $P_{V}$. In locations 63 to 68 of Figure $4 \mathrm{a}, P_{V} / P_{\text {span }}$ is reduced by over 0.10 . Consequently, at most locations, the $P_{S}+P_{D}$ by the proposed decomposition are generally higher or at least equal to that by other decompositions. Several isolated pixels have positive $\tau_{D}$, which may correspond to the trees in urban areas. In airport, $P_{V} / P_{\text {span }}$ is lowered by more than 0.03 , so we could observe the evident elevation of $P_{S} . P_{D}$ by all three decompositions are approximately the same.

Figure 4. Horizontal axis is pixel's relative position along profile. (a-d) are the profiles along the blue lines in Figure $3 b ;(\mathbf{e}-\mathbf{h})$ are the profiles along the green lines in Figure $3 b$; $(\mathbf{j}-\mathbf{n})$ are the profiles along the red lines in Figure $3 \mathrm{~b} ;(\mathrm{a}, \mathrm{e}, \mathrm{j})$ are the profiles of $P_{V} / P_{\text {span }}$; $(\mathrm{b}, \mathrm{f}, \mathrm{k})$ are $P_{D} / P_{\text {span }} ;(\mathrm{c}, \mathrm{g}, \mathrm{m})$ are $P_{S} / P_{\text {span }}$. In above 9 subfigures, red lines are the results of van Zyl decomposition, green lines are the results of Wang, and blue lines are the results of the proposed method; $(\mathrm{d}, \mathrm{h}, \mathrm{n})$ are the $\tau$ profiles along the blue, green, and red lines in Figure $3 \mathrm{~b}$, respectively. In $(\mathrm{d}, \mathrm{h}, \mathrm{n})$, red line is $\tau_{D}$, green line is $\tau_{V}$, blue line is $\tau_{S}$.

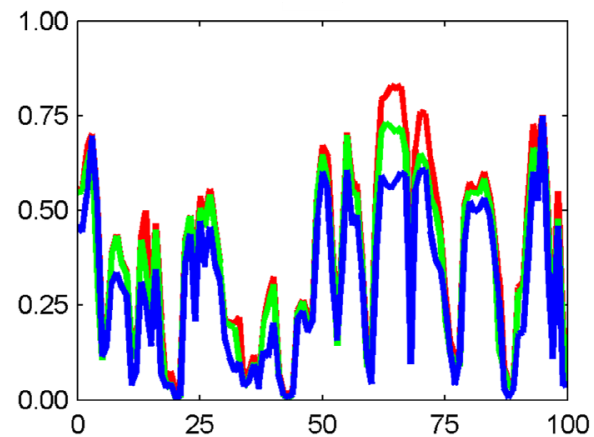

(a)

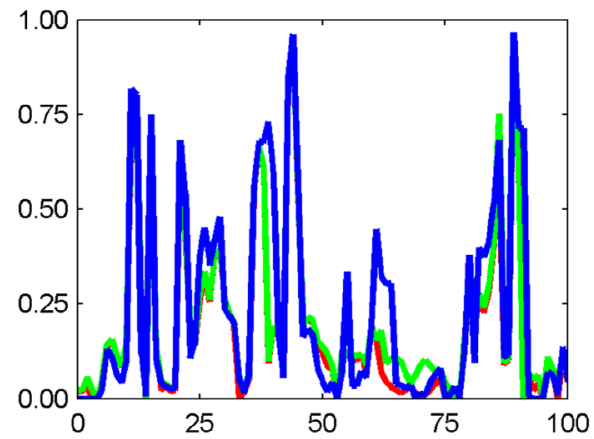

(c)

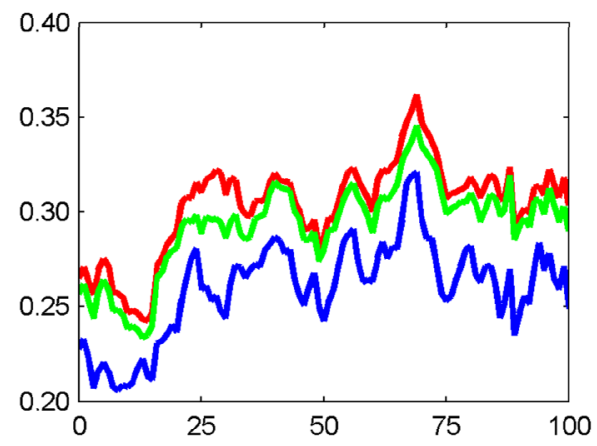

(e)

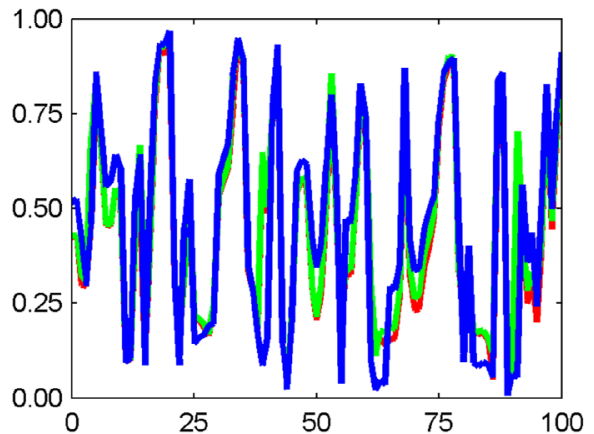

(b)

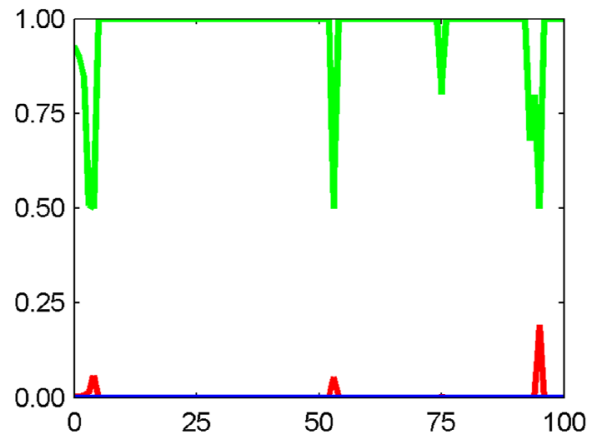

(d)

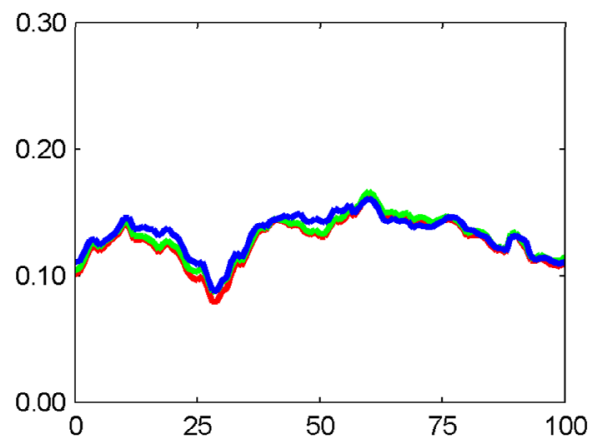

(f) 
Figure 4. Cont.

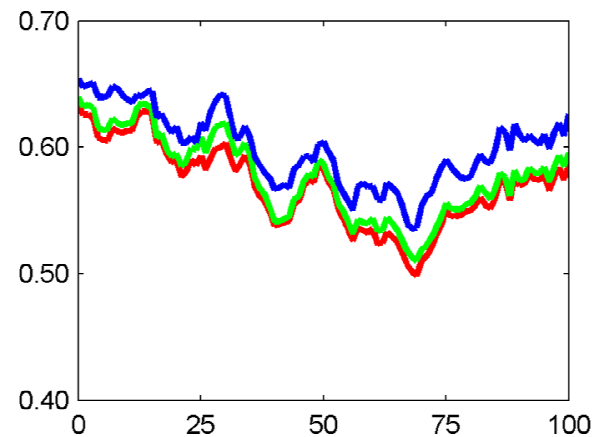

(g)

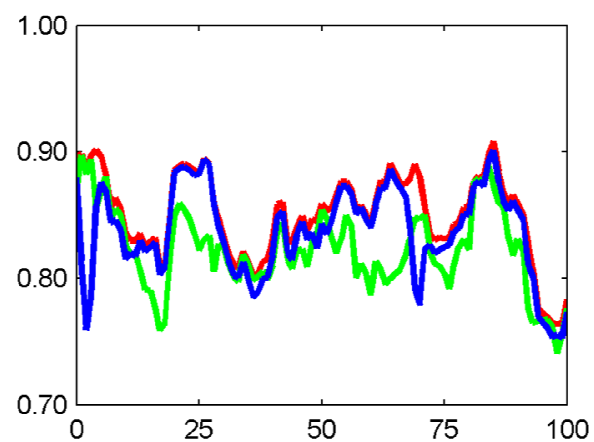

(j)

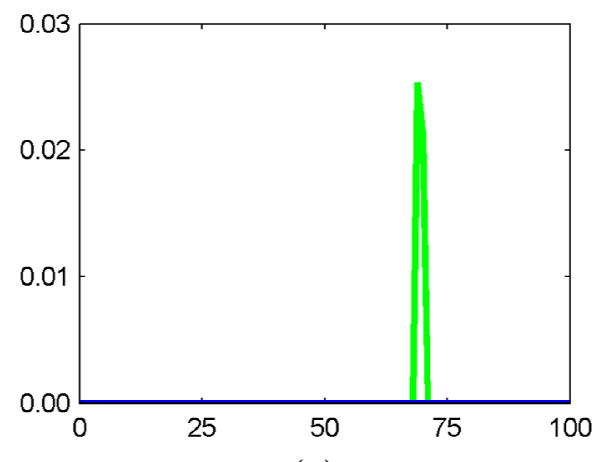

(m)

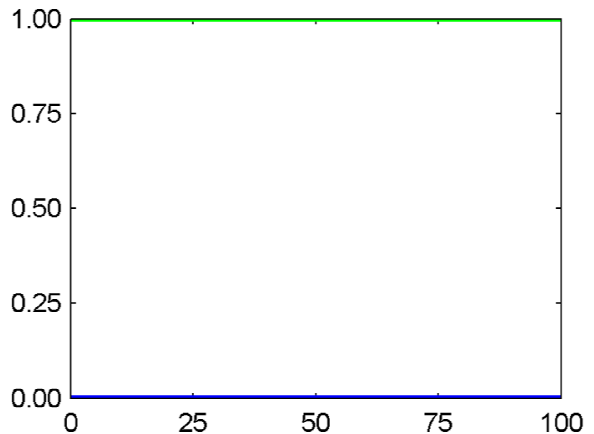

(h)

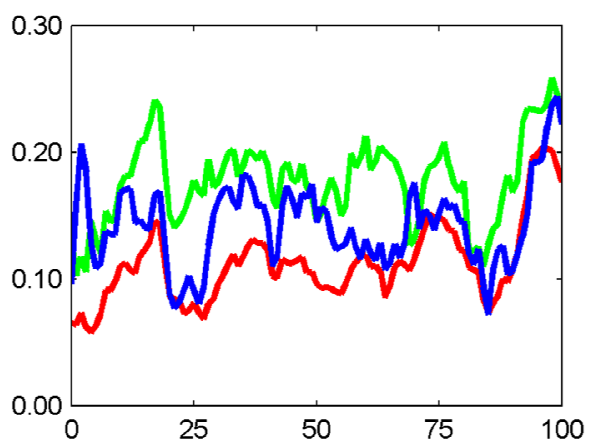

(k)

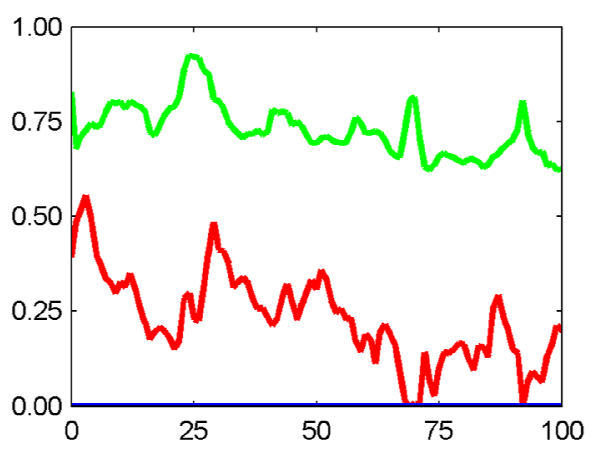

(n)

The dependency of decomposition results upon local incidence angles (short for incidence angle) was partly investigated. It is well known that SAR backscatter is influenced by land cover types and local incidence angle. Many land cover types reside in this study area, but it is difficult to select pixels with exactly the same land cover but different incidence angles. For simplicity, the authors just did this investigation for dense natural forests and lakes. First, dense natural forest and lake samples with the size $41 \times 41$ were selected in PolSAR image with the assistance of optical images. These samples could be roughly thought to be homogeneous inside. Next, the mean values of span-normalized powers of different components in diverse samples were plotted against their incidence angles (see Figure 5).

From Figure 5a, it seems the perspective that volume scattering dominates in dense natural forests only holds when the incidence angle is larger than a value, for example, $18^{\circ}$. When the incidence angle is medium or large, $P_{V} / P_{\text {span }}$ can be over 0.75 and double-bounce is much stronger than surface scattering. However, if the incidence angle is smaller than a value, like $26^{\circ}$, with the decreasing of incidence angle, $P_{V} / P_{\text {span }}$ drops quickly, at the same time, $P_{S} / P_{s p a n}$ goes up fast and surpasses $P_{D} / P_{\text {span. }}$. If the incidence angle is smaller than $10^{\circ}, P_{S} / P_{\text {span }}$ could be even larger than $P_{V} / P_{\text {span }}$. 
Figure 5. The relationship between normalized component powers and incidence angles. (a) Dense natural forest data; (b) Lake data. In both sub-figures, red line stands for $P_{D} / P_{\text {span }}$, green line stands for $P_{V} / P_{\text {span }}$, and blue line stands for $P_{S} / P_{\text {span }}$.

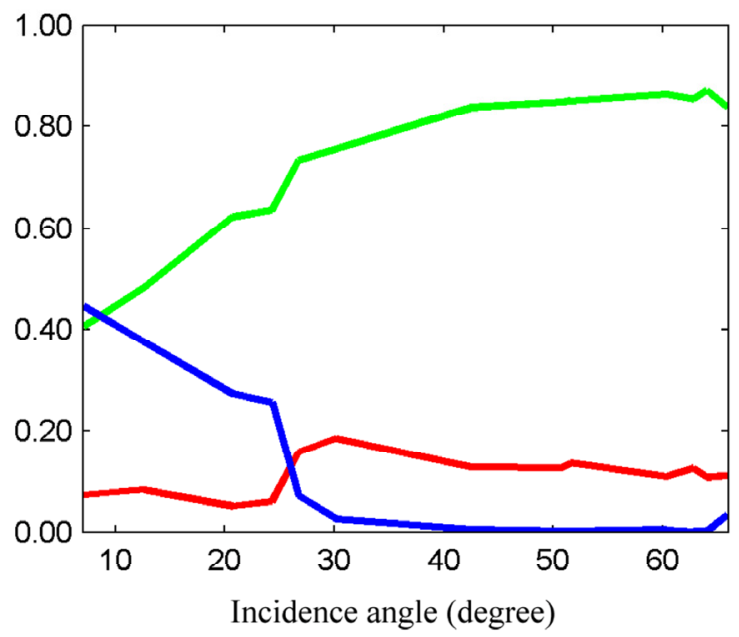

(a)

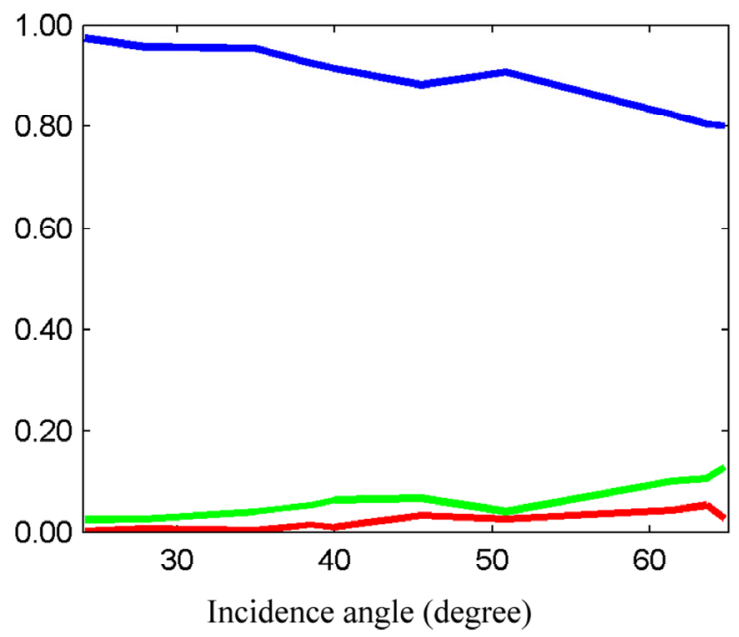

(b)

To understand above facts, the authors treat the volume scattering caused by canopies to be approximately isotropic [43], thus, when other factors are fixed, the volume scattering power does not vary much when incidence angle changes. However, the ordinary ground or soil is not isotropic. The smaller incidence angles the stronger surface scattering power; the larger incidence angles the weaker surface scattering power. In forest, double-bounce scattering is usually caused by ground-trunk structure. When the incidence angle is large, although the first scattering occurring at ground is not so strong, the microwave backscattered by the tree bark could be relatively strong due to the smooth bark and small incidence angle of the second scattering. On the contrary, when the incidence angle is small, the second scattering occurring at bark has very large incidence angle, possibly making the backscattered microwave be weak in comparison to surface scattering. Here, we summarize that, with the decreasing of incidence angle, $P_{S}, P_{\text {span }}$, and $P_{S} / P_{\text {span }}$ gradually increases; $P_{V} / P_{\text {span }}$ drops which is mainly due to the increasing of $P_{\text {span }}$.

Evidently, lakes are dominated by surface scattering, which is supported by the decomposition results in Figure 5b. No matter the incidence angle is small or large, $P_{S} / P_{\text {span }}$ is always much larger than $P_{D} / P_{\text {span }}$ and $P_{V} / P_{\text {span }}$. A basic trend is the larger incidence angle the smaller $P_{S} / P_{\text {span }}$.

Cheng [38] simulated PolSAR data with Bragg scatterer and incoherent scattering models. He observed such trend for surface scattering-dominated samples. He also found that the larger incidence angle the larger $T_{33} / P_{\text {span }}$. Since in most lake pixels, volume scattering and helix scattering explain all cross-polarized power, and $\tau_{V}=1$, we could easily infer that $P_{V} / P_{\text {span }}$ increases with $T_{33} / P_{\text {span }}$ or incidence angle.

\section{Discussion}

Experiment revealed the dependence of the decomposition results on land covers. Analytically explaining why these results are like this is challenging because many parameters are involved, so the authors show the relationship between $\tau_{V}$ and $P_{0}, P_{1}, P_{X}$ for four pixels, one is in airport and the 
other three are in forest (see Figure 6). Please note that if $P_{V_{-} \max }=P_{1}$, then $P_{X}=0$; if $P_{V_{-} \max }=P_{0}$, then $P_{X}>0$.

Figure 6. The relationship between $\tau_{V}$ and $P_{0}, P_{1}, P_{X}$. (a) A pixel in airport; (b-d) are pixels in forest. In all sub-figures, red line stands for $P_{0} / P_{s p}$, green line stands for $P_{1} / P_{s p}$, and blue line stands for $P_{X} / P_{s p}$, where $P_{s p}$ is the span of [T $\left.T_{O A C_{-} n o h}\right]$.

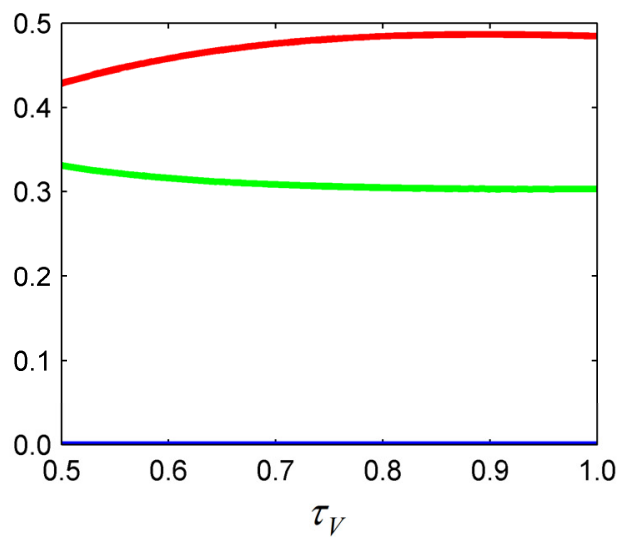

(a)

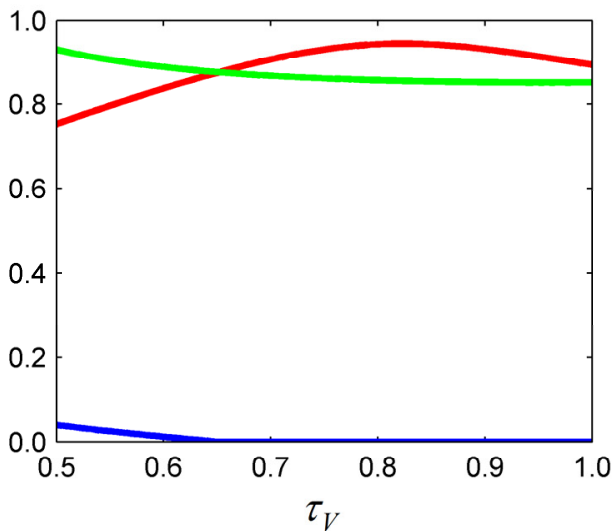

(c)

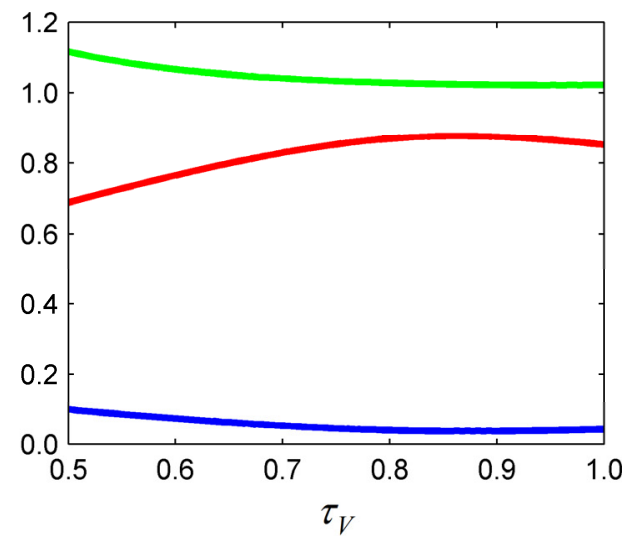

(b)

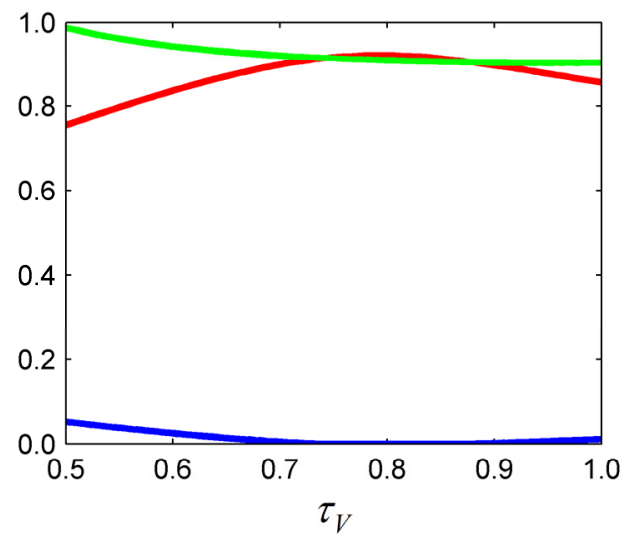

(d)

From the sub-figures in Figure 6, we could know $P_{1}$ is a monotonous decreasing function of $\tau_{V}$. The relationship between $P_{0}$ and $\tau_{V}$ is more complex: $P_{0}$ first monotonously increases with $\tau_{V}$ until reaches a peak at $\tau_{V}=\tau_{V_{-} 0}$, then monotonously decreases with $\tau_{V}$.

The line of $P_{0}$ and the line of $P_{1}$ are possible to have zero (see Figure 6a,b), one (see Figure 6c) or two (see Figure $6 \mathrm{~d}$ ) cross points. When these two lines do not intersect, then the situations are simple. It is observed that land covers dominated by surface scattering and double-bounce often have $A_{33} / P_{s p}<0.15$, or even $A_{33} / P_{s p}<0.10$, where $P_{s p}$ is the span of $\left[T_{O A C_{-} n o h}\right]$. For the same $\tau_{V}, P_{1}$ is always smaller than $P_{0} \quad$ (see Figure 6a), then $P_{V_{-} \max }=P_{1}$. Finally, $\tau_{V_{-} \operatorname{maxpv}}=0.5<\tau_{V_{-} \text {minpx }}=1.0$, $P_{V_{-} \text {max _maxpv }}=4 A_{33} /(1-g(0.5))>P_{V_{-} \text {max } \_ \text {minpx }}=4 A_{33}$. But as observed in UAVSAR data, dense natural forests usually show $A_{33} / P_{s p}>0.20$. If $A_{33} / P_{s p a n}>0.25$, then $P_{1} \geq 4 A_{33}>P_{s p}$, so the $P_{1}$ line and $P_{0}$ line never intersect, $P_{V_{-} \max }=P_{0}$ (see Figure 6b). In this case, $\tau_{V_{-} \operatorname{maxpv}}=\tau_{V_{-} 0}<\tau_{V_{-} \operatorname{minpx}}, P_{V_{-} \max \_ \text {maxpv }}>P_{V_{-} \max \_ \text {minpx }}$. Please remember that the $P_{0}$ line and $P_{1}$ line may do not intersect even when $A_{33} / P_{s p} \leq 0.25$. Some forest pixels have $P_{0}$ line and $P_{1}$ line intersected with one or two cross points. When there is one cross point (see Figure 6c), assume the cross 
point is $\left(\tau_{V_{-} 1}, P_{V_{-} \max \_1}\right)$, then in $\left[0.50, \tau_{V_{-} 1}\right], P_{V_{-} \max }=P_{0}$; in $\left[\tau_{V_{-} 1}, 1.0\right], P_{V_{-} \max }=P_{1}$. Finally, $\tau_{V_{-} \operatorname{maxpv}}=\tau_{V_{-} 1}<\tau_{V_{-} \operatorname{minp} x}=1.0, P_{V_{-} \text {max } \_ \text {maxpv }}=P_{V_{-} \text {max } \_1}>P_{V_{-} \text {max } \_ \text {minp }}=4 A_{33}$. When there are two cross points, assume the two cross points are $\left(\tau_{V_{-} 1}, P_{V_{-} m a x}{ }_{-1}\right)$ and $\left(\tau_{V_{-} 2}, P_{V_{-}}\right.$max $\left.\_2\right)$, and $\tau_{V_{-} 2}>\tau_{V_{-} 1}$, then in $\left[0.50, \tau_{V_{-} 1}\right]$ and $\left[\tau_{V_{-} 2}, 1.0\right], P_{V_{-} \max }=P_{0}$; in $\left[\tau_{V_{-} 1}, \tau_{V_{-}}\right], P_{V_{-} \max }=P_{1}$. Finally, $\tau_{V_{-} \text {maxpv }}=\tau_{V_{-} 1}<\tau_{V_{-} \text {minpx }}=\tau_{V_{-} 2}, P_{V_{-} \text {max } \_ \text {maxpv }}=P_{V_{-} \text {max } \_1}>P_{V_{-} \text {max } \_ \text {minpx }}=P_{V_{-} \text {max } \_2}$.

Why $P_{V}$ by the proposed decomposition is lower than that by van Zyl decomposition has been proven in previous sections. When it comes to the comparison between the proposed method and Wang decomposition, quantitatively explaining is much more difficult because Wang decomposition computes volume scattering parameters without RSA which is equal to solving cubic equations. Cheng [38] proved that for a fixed $\left[T_{V}\right], P_{V}$ computed without RSA is usually smaller than that computed with RSA. As a result, we are not sure whether $P_{V}$ computed without RSA and with maximum $P_{V}$ criterion is larger, or $P_{V}$ computed with RSA and minimum $P_{V}$ criterion is larger. That explains the situations of forest. For pixels dominated by surface scattering and double-bounce scattering, Wang decomposition commonly gives $\tau_{V}=0.5$, on the contrary, the proposed method has $\tau_{V}=1.0$. According to Equation (21), to explain the same amount of cross-polarized power, $P_{V}$ by Wang decomposition needs to be larger than that by the proposed method.

With simulated data, Cheng [38] found the proposed decomposition works worst in areas dominated by double-bounce scattering. In surface scattering-dominated areas, the cross-polarized power is usually relatively low, so even if we think cross-polarized power is entirely from volume scattering, the given $P_{V}$ will not be large. For areas dominated by volume scattering, letting volume scattering explain the most cross-polarized power in theory is reasonable to a large degree. However, it was pointed out in [36,37] that $T_{33}$ of double-bounce scattering model may be comparable to that of volume scattering model. In other words, sometimes, double-bounce scattering produces significant cross-polarized power. Minimum $P_{X}$ criterion forces volume scattering to explain as much cross-polarized power as possible. Therefore, a proportion of explained cross-polarized power is probably from double-bounce scattering instead of volume scattering. In this sense, $P_{V}$ may be overestimated.

\section{Conclusions}

The main differences between the proposed method and van Zyl, Cui, Wang's NNED include: (1) helix scattering are introduced in our method; (2) our method use minimum $P_{X}$ criterion while the three NNED use maximum $P_{V}$ criterion; (3) sometimes the dominant ground scattering is described by incoherent and depolarizing models, so there is no remainder component. In the three NNED, ground scattering are all coherently modeled; (4) to describe ground scattering, Cui and Wang NNED use elemental scatterers with $S_{H V} \neq 0$, but our method utilizes elemental scatterers with $S_{H V}=0$.

Negative component power is completely avoided in the proposed decomposition. In the experiment done by Cheng [38] with simulated data, he found that compared with van Zyl decomposition, the proposed method is capable of partly lessening volume scattering overestimation, which is mainly achieved by introducing helix scattering, utilizing minimum $P_{X}$ criterion, and performing two-component fitting to $\left[T_{O A C_{-} n o h}\right]$. But Cheng [38] also emphasized that minimum $P_{X}$ criterion could not fully eliminate $P_{V}$ overestimation in that volume scattering probably cannot explain the 
most cross-polarized power in theory. Ground scattering also contributes to cross-polarized power. The proposed method usually better estimates the power of each component than van Zyl, Wang and Cui decomposition. One significant advantage of the proposed method is, when volume scattering and helix scattering cannot explain all cross-polarized power, the dominant ground scattering is modeled by depolarizing models, so that its orientation angle randomness could be obtained. In the proposed decomposition, all cross-polarized power is explained by models with solid physical meanings.

How to utilize the power, complex scattering coefficients, orientation angle randomness of different components given by the proposed method for the applications like land cover mapping, scattering mechanism classification, understory mapping, or surface roughness and soil moisture estimation, needs more research. Another future research direction is proposing a decomposition that computes volume scattering parameters without RSA to utilize $T_{13}$ in $\left[T_{O A C_{-} n o h}\right]$.

\section{Acknowledgments}

This work was supported by the National Key Fundamental Research Plan of China (973) under Grant 2012CB719906. The authors would like to thank UAVSAR team in Jet Propulsion Laboratory, NASA for processing and providing UAVSAR data and the reviewers for reviewing this paper.

\section{Author Contributions}

Xiaoguang Cheng is the main proposer of the method in this paper as well as the chief author of this paper. Wenli Huang made great contribution to the improvement of language and writing of this paper. Jianya Gong provided much guidance to Xiaoguang Cheng in experiment design.

\section{Conflicts of Interest}

The authors declare no conflict of interest.

\section{References}

1. Ouchi, K. Recent trend and advance of synthetic aperture radar with selected topics. Remote Sens. 2013, 5, 716-807.

2. Freeman, A.; Durden, S.L. A three-component scattering model to describe polarimetric SAR data. Proc. SPIE 1992, 1748, 213-225.

3. Freeman, A.; Durden, S.L. A three-component scattering model for polarimetric SAR data. IEEE Trans. Geosci. Remote Sens. 1998, 36, 963-973.

4. Yamaguchi, Y.; Moriyama, T.; Ishido, M.; Yamada, H. Four-component scattering model for polarimetric SAR image decomposition. IEEE Trans. Geosci. Remote Sens. 2005, 43, 1699-1706.

5. An, W.; Cui, Y.; Yang, J. Three-component model-based decomposition for polarimetric SAR data. IEEE Trans. Geosci. Remote Sens. 2010, 48, 2732-2739.

6. Arii, M.; van Zyl, J.J.; Kim, Y. Adaptive model-based decomposition of polarimetric SAR covariance matrices. IEEE Trans. Geosci. Remote Sens. 2011, 49, 1104-1113. 
7. Van Zyl, J.J.; Arii, M.; Kim, Y. Model-based decomposition of polarimetric SAR covariance matrices constrained for nonnegative eigenvalues. IEEE Trans. Geosci. Remote Sens. 2011, 49, 3452-3459.

8. Cui, Y.; Yamaguchi, Y.; Yang, J.; Park, S.-E.; Kobayashi, H.; Singh, G. Three-component power decomposition for polarimetric SAR data based on adaptive volume scatter modeling. Remote Sens. 2012, 4, 1559-1572.

9. Singh, G.; Yamaguchi, Y.; Park, S.E. General four-component scattering power decomposition with unitary transformation of coherency matrix. IEEE Trans. Geosci. Remote Sens. 2013, 51, 3014-3022.

10. Singh, G.; Yamaguchi, Y.; Park, S.E.; Cui, Y.; Kobayashi, H. Hybrid freeman/eigenvalue decomposition method with extended volume scattering model. IEEE Geosci. Remote Sens. Lett. 2013, 10, 81-85.

11. Chen, S.-W.; Wang, X.-S.; Xiao, S.-P.; Sato, M. General polarimetric model-based decomposition for coherency matrix. IEEE Trans. Geosci. Remote Sens. 2014, 52, 1843-1855.

12. Cui, Y.; Yamaguchi, Y.; Yang, J.; Kobayashi, H.; Park, S.E.; Singh, G. On complete model-based decomposition of polarimetric SAR coherency matrix data. IEEE Trans. Geosci. Remote Sens. 2014, 52, 1991-2001.

13. Wang, C.; Yu, W.; Wang, R.; Deng, Y.; Zhao, F. Comparison of nonnegative eigenvalue decompositions with and without reflection symmetry assumptions. IEEE Trans. Geosci. Remote Sens. 2014, 52, 2278-2287.

14. Freeman, A. Fitting a two-component scattering model to polarimetric SAR data from forests. IEEE Trans. Geosci. Remote Sens. 2007, 45, 2583-2592.

15. Jong-Sen, L.; Ainsworth, T.L.; Yanting, W. Generalized polarimetric model-based decompositions using incoherent scattering models. IEEE Trans. Geosci. Remote Sens. 2014, 52, 2474-2491.

16. Antropov, O.; Rauste, Y.; Hame, T. Volume scattering modeling in POLSAR decompositions: Study of ALOS PALSAR data over boreal forest. IEEE Trans. Geosci. Remote Sens. 2011, 49, 3838-3848.

17. Da Silva, A.; Paradella, W.; Freitas, C.; Oliveira, C. Evaluation of digital classification of polarimetric SAR data for iron-mineralized laterites mapping in the amazon region. Remote Sens. 2013, 5, 3101-3122.

18. Lönnqvist, A.; Rauste, Y.; Molinier, M.; Häme, T. Polarimetric SAR data in land cover mapping in boreal zone. IEEE Trans. Geosci. Remote Sens. 2010, 48, 3652-3662.

19. Lardeux, C.; Frison, P.L.; Tison, C.; Souyris, J.C.; Stoll, B.; Fruneau, B.; Rudant, J.P. Support vector machine for multifrequency SAR polarimetric data classification. IEEE Trans. Geosci. Remote Sens. 2009, 47, 4143-4152.

20. Lee, J.-S.; Grunes, M.R.; Pottier, E.; Ferro-Famil, L. Unsupervised terrain classification preserving polarimetric scattering characteristics. IEEE Trans. Geosci. Remote Sens. 2004, 42, 722-731.

21. Qi, Z.; Yeh, A.G.-O.; Li, X.; Lin, Z. A novel algorithm for land use and land cover classification using RADARSAT-2 polarimetric SAR data. Remote Sens. Environ. 2012, 118, 21-39.

22. Lee, J.-S.; Grunes, M.R.; Schuler, D.L.; Pottier, E.; Ferro-Famil, L. Scattering-model-based speckle filtering of polarimetric SAR data. IEEE Trans. Geosci. Remote Sens. 2006, 44, 176-187. 
23. Ballester-Berman, J.D.; Lopez-Sanchez, J.M. Applying the freeman-durden decomposition concept to polarimetric SAR interferometry. IEEE Trans. Geosci. Remote Sens. 2010, 48, 466-479.

24. Yajima, Y.; Yamaguchi, Y.; Sato, R.; Yamada, H.; Boerner, W.-M. Polsar image analysis of wetlands using a modified four-component scattering power decomposition. IEEE Trans. Geosci. Remote Sens. 2008, 46, 1667-1673.

25. Shi, J.; Lee, J.-S.; Chen, K.; Sun, Q. Evaluate usage of decomposition technique in estimation of soil moisture with vegetated surface by multi-temporal measurements. In Proceedings of the 2000 IEEE International Geoscience and Remote Sensing Symposium, Honolulu, HI, USA, 25-30 July 2000; Volume 3, pp. 1098-1100.

26. Hajnsek, I.; Jagdhuber, T.; Schön, H.; Papathanassiou, K.P. Potential of estimating soil moisture under vegetation cover by means of POLSAR. IEEE Trans. Geosci. Remote Sens. 2009, $47,442-454$.

27. Jagdhuber, T.; Schön, H.; Hajnsek, I.; Papathanassiou, K.P. Soil moisture estimation under vegetation applying polarimetric decomposition techniques. In Proceedings of the 4th International Workshop on Science and Applications of SAR Polarimetry and Polarimetric Interferometry, Frascati, Italy, 26-30 January 2009; ESA: Frascati, Italy, 2009; pp. 1-8.

28. Tello, M.; López-Martínez, C.; Mallorqui, J.J. A novel algorithm for ship detection in SAR imagery based on the wavelet transform. IEEE Geosci. Remote Sens. Lett. 2005, 2, 201-205.

29. Sato, R.; Takahashi, Y.; Yamaguchi, Y.; Yamada, H. stricken man-made object detection using scattering power decomposition with NNED and rotation of the covariance matrix. In Proceedings of the 2012 IEEE International Geoscience and Remote Sensing Symposium, Munich, Germany, 22-27 July 2012; pp. 5121-5124.

30. Yonezawa, C.; Watanabe, M.; Saito, G. Polarimetric decomposition analysis of ALOS PALSAR observation data before and after a landslide event. Remote Sens. 2012, 4, 2314-2328.

31. Arii, M.; van Zyl, J.J.; Kim, Y. A general characterization for polarimetric scattering from vegetation canopies. IEEE Trans. Geosci. Remote Sens. 2010, 48, 3349-3357.

32. Neumann, M. Remote Sensing of Vegetation Using Multi-Baseline Polarimetric Sar Interferometry: Theoretical Modeling and Physical Parameter Retrieval. Ph.D. Thesis, Université de Rennes 1, Rennes, France, 2009.

33. Lee, J.-S.; Ainsworth, T.L. The effect of orientation angle compensation on coherency matrix and polarimetric target decompositions. IEEE Trans. Geosci. Remote Sens. 2011, 49, 53-64.

34. Chen, S.-W.; Ohki, M.; Shimada, M.; Sato, M. Deorientation effect investigation for model-based decomposition over oriented built-up areas. IEEE Geosci. Remote Sens. Lett. 2013, 10, 273-277.

35. Neumann, M.; Ferro-Famil, L.; Pottier, E. A General model based polarimetric decomposition scheme for vegetated areas. In Proceedings of the 4th International Workshop on Science and Applications of SAR Polarimetry and Polarimetric Interferometry (ESRIN), Frascati, Italy, 26-30 January 2009.

36. Cheng, X.; Huang, W.; Gong, J. An unsupervised scattering mechanism classification method for polsar images. IEEE Geosci. Remote Sens. Lett. 2014, 11, 1677-1681.

37. Cheng, X.; Huang, W.; Gong, J. A decomposition-free scattering mechanism classification method for PoLSAR images with Neumann's model. Remote Sens. Lett. 2013, 4, 1176-1184. 
38. Cheng, X. Research of Model-Based Polarimetric Sar Decomposition Constrained for Nonnegative Eigenvalues. Ph.D. Thesis, Wuhan University, Wuhan, China, 2014.

39. Rosen, P.A.; Hensley, S.; Wheeler, K.; Sadowy, G.; Miller, T.; Shaffer, S.; Muellerschoen, R.; Jones, C.; Zebker, H.; Madsen, S. UAVSAR: A new NASA Airborne SAR system for science and technology research. In Proceedings of the 2006 IEEE Conference on Radar, Verona, NY, USA, 24-27 April 2006; pp. 22-29.

40. UAVSAR-Home. Available online: http://uavsar.jpl.nasa.gov/instrument.html (accessed on 4 September 2013).

41. Vertex: ASF's Data Portal. Available online: https://vertex.daac.asf.alaska.edu (accessed on 1 May 2012).

42. Lee, J.-S.; Wen, J.-H.; Ainsworth, T.L.; Chen, K.-S.; Chen, A.J. Improved sigma filter for speckle filtering of SAR imagery. IEEE Trans. Geosci. Remote Sens. 2009, 47, 202-213.

43. Cheng, X.; Pinto, N.; Gong, J. Terrain radiometric calibration of airborne UAVSAR for forested area. Geo-Spat. Inf. Sci. 2012, 15, 229-240.

\section{Appendix}

To simplify the computation of $S_{H H}$ and $S_{V V}$, we assume $S_{H H}=1$ and $S_{V V}=b_{1}+b_{2} j$ as that in Freeman-Durden decomposition [3]. Take them into $\left[T_{\text {Remainder } 2}\right]$, if $\operatorname{Im}\left(G_{12}\right) \neq 0$, we have

$$
\begin{aligned}
& \frac{L}{N}=\frac{\left(1+b_{1}\right)^{2}+b_{2}^{2}}{\left(1-b_{1}\right)^{2}+b_{2}^{2}}=\frac{G_{11}}{G_{22}+G_{33}}=k_{1} \\
& \frac{\operatorname{Re}(M)}{\operatorname{Im}(M)}=\frac{1-b_{1}^{2}-b_{2}^{2}}{2 b_{2}}=\frac{\operatorname{Re}\left(G_{12}\right)}{\operatorname{Im}\left(G_{12}\right)}=k_{2}
\end{aligned}
$$

The solutions of (A1) and (A2) are

$$
\begin{gathered}
b_{1}=\frac{\left(k_{1}-1\right)\left(\left(1+k_{2}^{2}\right)\left(1+k_{1}\right)-k_{2} \sigma\right)}{D-2 k_{1}} \\
b_{2}=\frac{\sigma-k_{1}\left(4 k_{2}+\sigma\right)}{D}
\end{gathered}
$$

and

$$
\begin{gathered}
b_{1}=\frac{\left(k_{1}-1\right)\left(\left(1+k_{2}^{2}\right)\left(1+k_{1}\right)+k_{2} \sigma\right)}{D-2 k_{1}} \\
b_{2}=-\frac{\sigma+k_{1}\left(4 k_{2}+\sigma\right)}{D}
\end{gathered}
$$

with

$$
\begin{gathered}
\sigma=2 \sqrt{k_{1}\left(k_{2}^{2}+1\right)} \\
D=\left(k_{1}^{2}+1\right)\left(k_{2}^{2}+1\right)-2 k_{1}\left(k_{2}^{2}-1\right)
\end{gathered}
$$

For the two solutions in Equations (A3) and (A4), the one whose $b_{2}$ sign is the same as $\operatorname{Im}\left(G_{12}\right)$ should be chosen. If $\operatorname{Im}\left(G_{12}\right)=0$, then $b_{2}=0, b_{1}$ is 


$$
b_{1}=\frac{\sqrt{k_{1}}-1}{\sqrt{k_{1}}+1}
$$

or

$$
b_{1}=\frac{\sqrt{k_{1}}+1}{\sqrt{k_{1}}-1}
$$

The two $b_{1}$ solutions in Equations (A7) and (A8) are the inverse of each other. Which one is better may be determined with the help of physical models, like Bragg model [26]. For example, for surface scattering, Bragg model predicts $\left|S_{V V}\right|<1$, so the smaller one of Equations (A7) and (A8) should be chosen.

(C) 2014 by the authors; licensee MDPI, Basel, Switzer land. This article is an open access article distributed under the terms and conditions of the Creative Commons Attribution license (http://creativecommons.org/licenses/by/3.0/). 